\title{
Melanotic Oncocytic Metaplasia of the Nasopharynx in the Patient with Suspicious Hemoptysis: Case Report
}

\author{
Taek Yoon Cheong, $\mathrm{MD}^{1}$, Han Seong Kim, $\mathrm{MD}, \mathrm{PhD}^{2}$, and Ick Soo Choi, $\mathrm{MD}, \mathrm{PhD}^{1}$ \\ ${ }^{I}$ Department of Otorhinolaryngology-Head and Neck Surgery and ${ }^{2}$ Pathology, Ilsan Paik Hospital, Inje University College of Medicine, \\ Goyang, Korea
}

\begin{abstract}
Melanotic oncocytic metaplasia (MOM) in the nasopharyngeal space is a very rare entity. Only 35 cases have been reported in the English literature, and most patients were East Asian males between 60 and 70 years of age. MOM presents as a brown or black lesion with slight elevation of the mucosa. These lesions are benign and defined as cellular enlargement with eosinophilic granular melanin-pigmented cytoplasm caused by mitochondrial accumulation. However, such presentation can lead physicians to misjudge MOM as a malignant lesion. Recently, we experienced a case of MOM of the nasopharynx. A 58-year-old woman was admitted to the internal medicine department with small-volume hemoptysis and referred to the ENT department for evaluation. She was a regular smoker without any medical history. Sinus endoscopy showed black pigmented lesions on both the torus tubaris and left posterior tonsillar pillar, with low bleeding risk. Excisional biopsy of the lesion was performed, and oncocytic metaplasia was confirmed pathologically. Hemoptysis showed spontaneous remission and no recurrence or other symptoms over 12 months of follow up. Melanotic oncocytic metaplasia in the nasopharynx should be clinically recognized to avoid misdiagnosis as a malignancy like melanoma.
\end{abstract}

KEY WORDS: Adenoma, Oxyphillic · Nasopharynx $\cdot$ Hemoptysis.

\section{INTRODUCTION}

First melanotic oncocytic metaplasia (MOM) of the nasopharynx was reported in 1995. ${ }^{1)}$ MOM can be misdiagnosed as early nasopharyngeal carcinoma or melanoma, due to its macroscopic appearance. However, MOM is clearly distinct from such diseases histologically. MOM comprises both oncocytic metaplasia and melanin pigmentation of the epithelium in the same gland. Oncocytes are big epithelial cells with an aplenty, deeply eosinophilic granular cytoplasm and a small, round, dark colored nucleus. Melanocytes found to coexist in MOM, is considered to be the source of melanin. ${ }^{2)}$ No atypia or mitotic activity found in MOM. Fontana-Masson staining is positive and highlights several dendritic melanocytes between the metaplastic and the surface epithelial cells. ${ }^{3)} \mathrm{MOM}$ is thought to be an age-related change since its incidence increases with age, and predominantly occurs in Asian population. ${ }^{4)}$

MOM of the nasopharynx is a very rare lesion, and only 35 cases were reported in English literature. Most lesions were asymptomatic and incidentally discovered during physical examination.

We present here a case of MOM of the nasopharynx, found in a patient with suspicious hemoptysis.

\section{CASE REPORT}

A 58-year-old woman was admitted to internal medicine department, presenting small volume hemoptysis for 5 days. She was a regular smoker $(10 /$ day $\times 20$ years $)$ without any

Received: July 30, 2020 / Revised: September 14, 2020 / Accepted: October 5, 2020

Address for correspondence: Ick Soo Choi, MD, PhD, Department of Otorhinolaryngology-Head and Neck Surgery, Ilsan Paik Hospital, Inje University College of Medicine, 170 Juhwa-ro, Ilsanseo-gu, Goyang 10380, Korea

Tel: +82-31-910-7114, Fax: +82-31-910-7747, E-mail: Leochoics@gmail.com 
medical history. Patient admitted and undergone computed tomography scan of lung. Since the radiologic evaluation at the internal medicine department showed no clinical signifi-
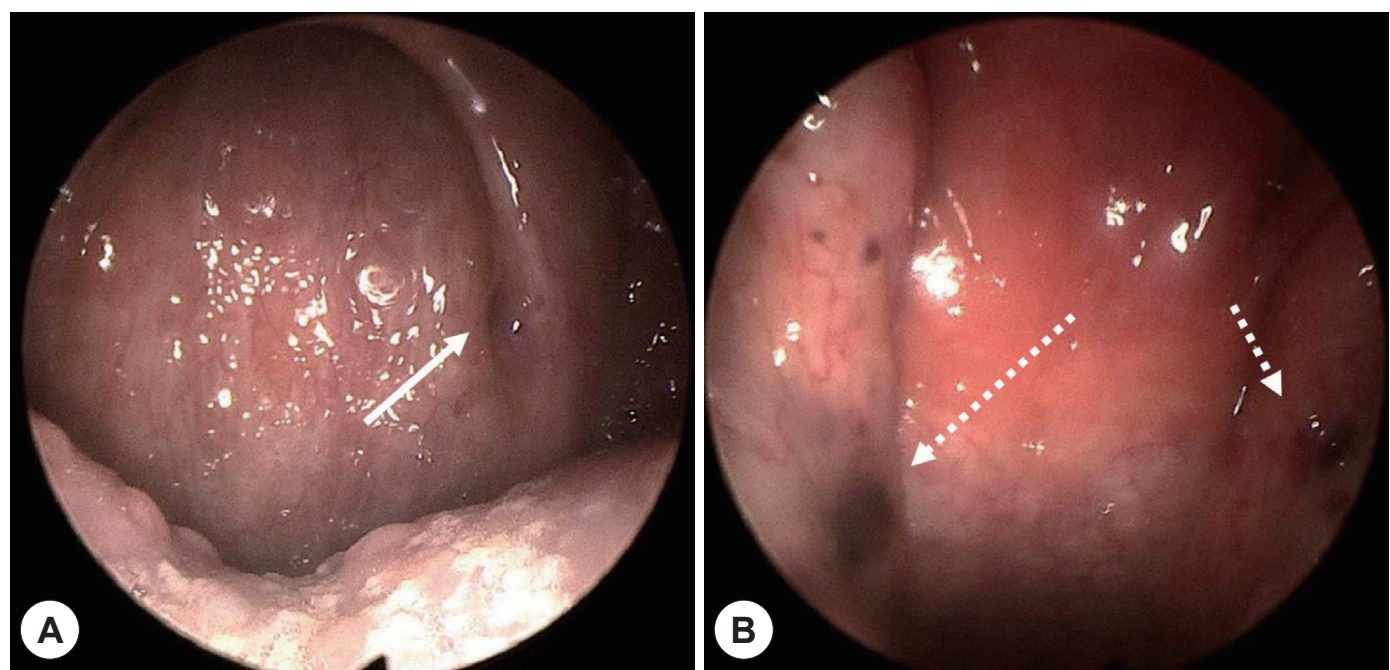

Fig. 1. Endoscopic findings. A: Endoscopic finding of melanotic oncocytic metaplasia on left anterior pharyngeal pillar (white arrow). B: Endoscopic finding of melanotic oncocytic metaplasia on both torus tubaris (white dotted arrows).
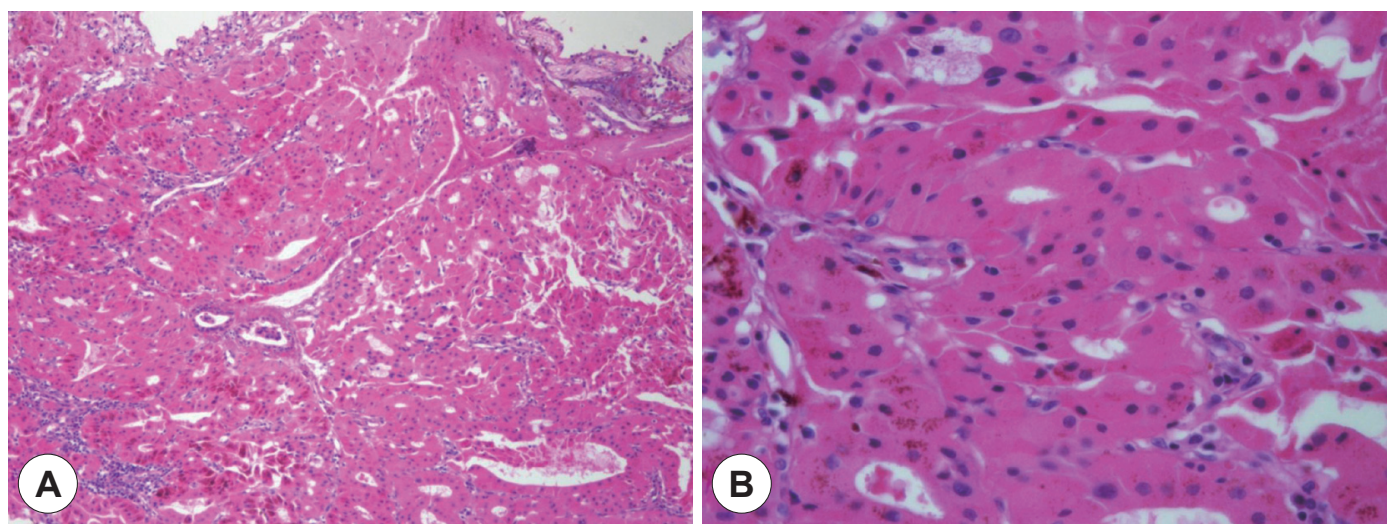

Fig. 2. Pathologic results of the case. A: Microscopic finding $(\times 100)$ shows deeply homogeneous eosinophilic epitheial cells, noted with lumen formation. B: Scattered melanin pigment granules are found in eosinophilic cytoplasm $(\times 400)$.
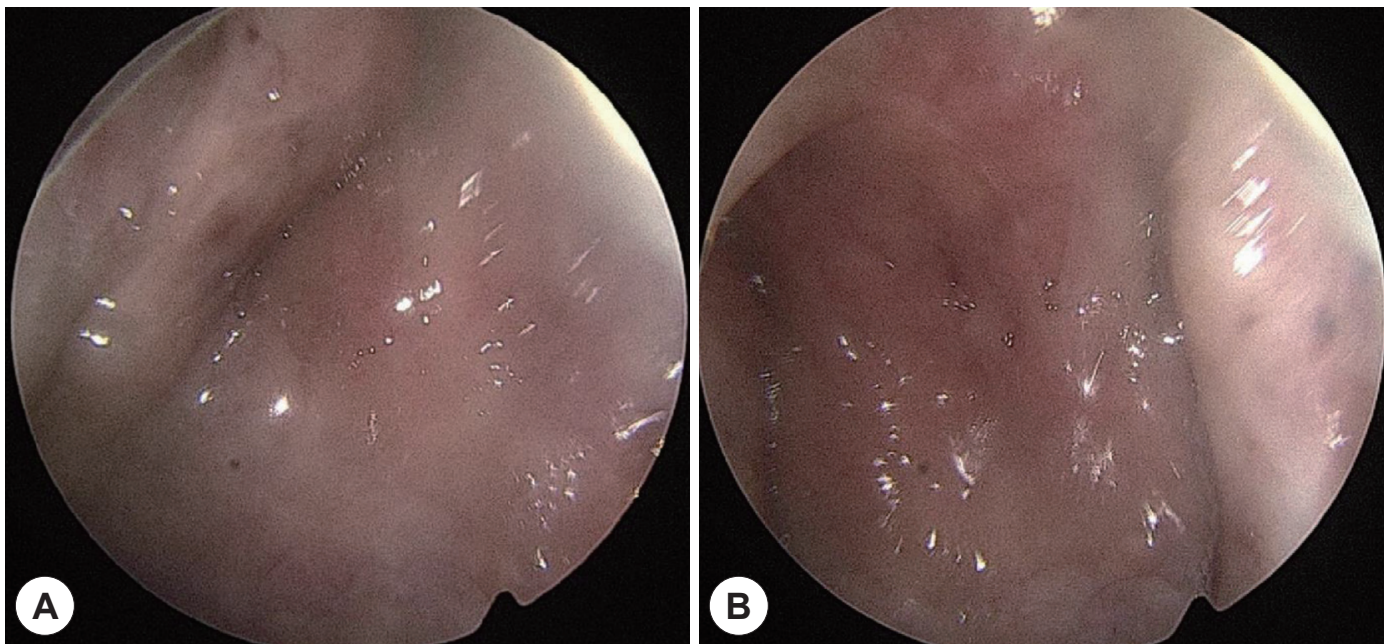

Fig. 3. Endoscopic findings after 12 months. A: Rt torus tubaris. B: Lt torus tubaris. 
mented lesions on both torus tubaris and left posterior ton- hemoptysis stopped spontaneously during the admission sillar pillar (Fig. 1), but bleeding from the lesion was unseen. period, surgical biopsy of the lesion was performed to rule Sinus mucosa and anatomy was intact otherwise. Though out possible malignancy. Five specimens were sent for he-

Table 1. Reported cases of melanotic oncocytic metaplasia of nasopharynx

\begin{tabular}{|c|c|c|c|c|c|c|}
\hline Case & Sex/age & Site & $\begin{array}{l}\text { Chief complaint } \\
\text { for visiting clinic }\end{array}$ & $\begin{array}{l}\text { Number } \\
\text { of lesions }\end{array}$ & $\begin{array}{l}\text { Smoking } \\
\text { history }\end{array}$ & Author/year \\
\hline 1 & $M / 67$ & EO & Otitis media & Single & Unknown & Shek TW, $1995^{1}$ \\
\hline 2 & $M / 63$ & EO & Tinnitus & Single & Unknown & Shek TW, 1995 \\
\hline 3 & $M / 70$ & Bilateral EO & Tinnitus & Multiple & Unknown & Xue WC, $1999^{5}$ \\
\hline 4 & $M / 64$ & $\begin{array}{l}\text { Bilateral EO, left suprapharynx, } \\
\text { and nasal cavity }\end{array}$ & Throat discomfort & Multiple & Unknown & $\begin{array}{l}\text { Hirakawa E, } \\
1999^{6}\end{array}$ \\
\hline 5 & $M / 62$ & $\begin{array}{l}\text { Left EO and bilateral torus } \\
\text { tubaris }\end{array}$ & Discomfort in Lt ear & Multiple & Unknown & Takano K, $2004^{7}$ \\
\hline 6 & $M / 69$ & Left nasopharynx & Foreign body swallowing & Multiple & Unknown & Kurihara K, $1997^{8}$ \\
\hline 7 & $M / 80$ & $\begin{array}{l}\text { Right nasal cavity and } \\
\text { pharynx }\end{array}$ & Hoarseness & Multiple & $10 /$ day $\times 50 y r$ & Sakaki M, $2004^{9}$ \\
\hline 8 & $M / 69$ & Left EO & Hoarseness & Single & $40 /$ day $\times 60 y r$ & Sakaki M, 2004 \\
\hline 9 & $M / 74$ & Left nasopharynx & Rhinorrhea & Single & Unknown & Sakaki M, 2004 \\
\hline 10 & $\mathrm{~F} / 74$ & Right EO & Throat discomfort & Multiple & Unknown & Sakaki M, 2004 \\
\hline 11 & $M / 68$ & Nasopharynx & None & Single & Unknown & Sakaki M, 2004 \\
\hline 12 & $M / 65$ & Right EO & Hemoptysis & Single & Unknown & Sakaki M, 2004 \\
\hline 13 & $M / 63$ & Left EO & Epistaxis & Single & Unknown & Sakaki M, 2004 \\
\hline 14 & M/- & Bilateral nasopharynx & $\begin{array}{l}\text { None (examined to find origin } \\
\text { site of metastatic carcinoma) }\end{array}$ & Multiple & Unknown & Liao C, $2005^{10}$ \\
\hline 15 & $M / 79$ & Right EO & Otitis media & Single & $20 /$ day $\times 40 y r$ & Lui PC, $2004^{11}$ \\
\hline 16 & $M / 58$ & Bilateral nasopharynx & Epistaxis & Multiple & Unknown & $\mathrm{Li} Y, 2010^{12}$ \\
\hline 17 & $M / 73$ & Bilateral torus tubaris & Nasal congestion & Multiple & Non-smoker & Kondo, $2010^{13}$ \\
\hline 18 & $M / 72$ & Bilateral torus tubaris & $\begin{array}{l}\text { Headache and hearing } \\
\text { impairment }\end{array}$ & Multiple & $40 /$ day $\times 50 y r$ & $J Y ~ N a, 2012^{14}$ \\
\hline 19 & $M / 71$ & $\begin{array}{l}\text { Left torus tubaris and } \\
\text { soft palate }\end{array}$ & Hoarseness & Multiple & $20 /$ day $\times 40 y r$ & JY Na, 2012 \\
\hline 20 & $M / 51$ & Right torus tubaris & Tongue pain & Multiple & Unknown & JY Na, 2012 \\
\hline 21 & $M / 63$ & Nasopharynx & Epistaxis & Multiple & $40 /$ day $\times 40 y r$ & Chang, $2014^{15}$ \\
\hline 22 & $M / 57$ & Right torus tubaris & $\begin{array}{l}\text { None (examined to find origin } \\
\text { site of metastatic carcinoma) }\end{array}$ & Multiple & $30 /$ day $\times 40 y r$ & $\begin{array}{l}\text { Shogo Tajima, } \\
20152\end{array}$ \\
\hline 23 & $\mathrm{~F} / 70$ & EO & $\begin{array}{l}\text { Routine gastrointestinal } \\
\text { endoscopy }\end{array}$ & Multiple & Unknown & Uehara K, $2015^{16}$ \\
\hline 24 & $\mathrm{~F} / 61$ & Left EO & Hoarseness & Single & Unknown & Uehara K, 2015 \\
\hline 25 & $M / 74$ & Bilateral nasopharynx & Hoarseness & Multiple & Unknown & Uehara K, 2015 \\
\hline 26 & $M / 57$ & Right nasopharynx & Nasal obstruction & Single & Smoker & Li J, $2019^{17}$ \\
\hline 27 & $M / 61$ & Left nasopharynx & Neck mass & Single & Smoker & Li J, 2019 \\
\hline 28 & $M / 69$ & Left nasopharynx & Rhinorrhea & Multiple & Smoker & Li J, 2019 \\
\hline 29 & $M / 56$ & Left EO & Epistaxis & Single & Smoker & Li J, 2019 \\
\hline 30 & $M / 58$ & Right nasopharynx & Facial palsy & Single & Smoker & Li J, 2019 \\
\hline 31 & $M / 52$ & Left EO & Tinnitus & Multiple & Smoker & Li J, 2019 \\
\hline 32 & $\mathrm{~F} / 77$ & Left nasopharynx & Hoarseness & Single & Smoker & Li J, 2019 \\
\hline 33 & $M / 59$ & Left nasopharynx & Nasal obstruction & Single & Smoker & Li J, 2019 \\
\hline 34 & $M / 59$ & Left EO & Tinnitus & Single & Smoker & Li J, 2019 \\
\hline 35 & $M / 75$ & Right EO & Epistaxis & Multiple & $30 /$ day $\times 50 \mathrm{yr}$ & Chen HY, 2020 ${ }^{18}$ \\
\hline 36 & $F / 53$ & $\begin{array}{l}\text { Left posterior tonsillar pillar, } \\
\text { both torus tubaris }\end{array}$ & Hemoptysis & Multiple & $10 /$ day $\times 20 y r$ & $\begin{array}{l}\text { Present case, } \\
2020\end{array}$ \\
\hline
\end{tabular}


matoxylin and eosin(H\&E) staining. Microscopically, homogenous oncocytic cells with melanin pigment granules in their cytoplasm were found, and melanotic oncocytic metaplasia was pathologically confirmed (Fig. 2). Hemoptysis stopped spontaneously. The patient discharged without complications and didn't presented hemoptysis or other symptoms during 12 months follow up. Endoscopic finding showed no further growth or advancement of previous lesion (Fig. 3).

\section{DISCUSSION}

Oncocytic change found in the upper respiratory tract is an unusual finding. Melanotic variation of oncocytic metaplasia is an extremely anomalous condition, presented as a small, brown to black mucosal lesion. ${ }^{3)}$ To our knowledge, only 35 cases have been reported in the English literature, and our case is the 36th (Table 1). All reported cases were elderly Asian, supporting the possibility of ethnic background as a predisposing factor of MOM. MOM predominantly occurred in males (31/36), and most of patients were long time smoker $(18 / 19)$, supporting the hypothesis that smoking might be a predisposing factor for MOM. The male predominance of patients supports this hypothesis also, since male smokers are more frequent among Asian population. 16 cases showed single lesion while other 20 cases presented multiple lesions in nasopharynx. The presented patient was an Asian female with multiple lesions, and smoking history was relatively short compared to other 17 previously reported cases with smoking history.

Pathological findings of the patient in H\&E stain showed oncocytic metaplasia and scattered melanin pigment granules. Atypia or mitotic figures were not found. Oncocytic cells are epithelial cells, characterized by abundant eosinophilic cytoplasm. Yet, oncocytic metaplasia is uncommon finding in upper respiratory tract. The exact origin of the melanin pigment in MOM is still in question. However, previous studies demonstrated that Fontana-Masson stain, S-100 protein immunostain and human melanoma black-45 immunostain revealed dendritic melanocytes stretching through the epithelial cells of the glands in $\mathrm{MOM}^{2}{ }^{29)}$ supporting that melanin pigment may be derived from the adjoining melanocytes through their stretching dendrites. ${ }^{614)}$

Most of the cases were found incidentally with other symptoms such as tinnitus, hoarseness, epistaxis, rhinorrhea, and hemoptysis. Some symptoms, such as ear discomfort at the same side of MOM near eustachian opening, seemed to be related with the lesion, while other symptoms like hoarseness seemed almost independent from the lesion.

It is unclear whether MOM can cause hemoptysis or not. One case with hemoptysis was reported previously, ${ }^{9)}$ but it's clinical course or relation between the lesion was not described. Three cases with epistaxis had been reported also. During the endoscopic biopsy of presented case, the lesions didn't show hemorrhagic features, supporting irrelevance between hemoptysis and the lesion. Minor bronchopneumonia could have been the origin of hemoptysis in this case, but was not clearly identified.

Clinically, carcinoma or melanoma may show similar appearance with MOM during the endoscopic examination. Differential diagnosis between those entities are straightforward histologically, so physicians encountered such lesions should try biopsy. Endoscopic findings isn't sufficient to distinguish between MOM and nasopharyngeal malignancy since nasopharyngeal malignancy may shows various forms including submucosal lesions.

It is known that MOM follows a benign clinical path. Simple excision is a suitable treatment for MOM. ${ }^{2)}$ No abnormal bleeding or surgical difficulty was found in operation of this case. Since no recurrence or advancement of the lesion has been reported in the literatures, diagnosed MOM can be observed without any further treatment.

In conclusion, we report the 36 th cases of melanotic oncocytic metaplasia in the nasopharynx. As a benign imitator of malignant lesion, MOM should be always taken into consideration in examination of nasopharynx.

\section{REFERENCES}

1) Shek TWH, Luk ISC, Nicholls JM, Fok KO. Melanotic oncocytic metaplasia of the nasopharynx. Histopathology 1995;26(3):273-5.

2) Tajima S, Ohkubo A, Yoshida M, Koda K, Nameki I. Melanotic oncocytic metaplasia of the nasopharynx: a case report with a focus on immunohistochemical analyses and literature review. International Journal of Clinical and Experimental Pathology 2015;8(2): 2103-10.

3) Antonio C, Pieter J, Nina G, Alessandro F. Pathology of the Head and Neck. 2nd ed. Berlin, Heidelberg: Springer;2016.

4) Mills S. Histology for Pathologists. Philadelphia: Lipincott Williams \& Wilkins;2012.

5) Xue WC, Hui YZ. Melanotic oncocytic metaplasia of the nasopharynx. Histopathology 1999;35(5):481-2.

6) Hirakawa E, Miki H, Ohmori M, Kobayashi S, Haba R, Nagai Y. Melanin pigmented oncocytic metaplasia of the nasopharynx. Virchows Archiv 1999;434(5):455-7.

7) Takano KI, Sato J, Shirasaki H, Yamazaki N, Hoki K, Himi T. Mel- 
anin pigmented oncocytic metaplasia of the nasopharynx. Auris Nasus Larynx 2004;31(2):161-3.

8) Kurihara K, Nakagawa K. Pigmented variant of benign oncocytic lesion of the pharynx. Pathology International 1997;47(5):315-7.

9) Sakaki M, Shek TWH, Hirokawa M, Kashima K, Daa T, Gamachi A, et al. Melanotic oncocytic metaplasia of the nasopharynx: a report of seven cases and review of the literature. Virchows Archiv 2004;444 (4):345-9.

10) Liao CT, Kuo TT. Melanotic Oncocytic Metaplasia of the Nasopharynx. International Journal of Surgical Pathology 2005;13(3):279.

11) Lui PCW, Chan ABW, Chan KF, Choi CH, Tse GMK. Melanocytic and non-melanocytic oncocytic metaplasia of the nasopharynx. Pathology 2004;36(5):504-5.

12) Li Y, Lu ZH, Lü W, Chen J. Images for diagnosis. Melanotic oncocytic metaplasia of nasopharynx: a case report with review. Chin Med J (Engl) 2010;123(9):1230-2.

13) Kondo T, Mori K, Oka S, Morinaka S. Melanotic oncocytic meta- plasia of the nasopharynx as a benign mimicker of malignant melanoma: a case report. Diagnostic Pathology 2010;5:5.

14) Na JY, Kim YH, Choi YD, Lee JS. Melanotic oncocytic metaplasia of the nasopharynx: a report of three cases and review of the literature. Korean Journal of Pathology 2012;46(2):201-4.

15) Chang IW, Wang CC, Liu KW, Lan CH, Hung CH. Melanotic oncocytic metaplasia of the nasopharynx. Polish Journal of Pathology 2014; 65(2):162-5.

16) Uehara K, Usami Y, Imai Y, Shimizu M. Melanotic oncocytic metaplasia of the nasopharynx. Pathology International 2015;65(3):144-7.

17) Li JJX, Ng JKM, Chan ABW. Clinicopathological features of melanotic and non-melanotic oncocytic lesions of the nasopharynx. Pathology 2019;51(6):600-4.

18) Chen HY, Gule MF, Chang IW. Melanotic oncocytic metaplasia of the nasopharynx: a case report with review of literature. Ear Nose Throat J 2020:145561320907427. 